\title{
Plant-derived angiogenin fusion protein's cytoprotective effect on trabecular meshwork damage induced by Benzalkonium chloride in mice
}

\author{
Jae Hoon Jeong ${ }^{1,2,3}$, Soo Jin Lee ${ }^{4}$, Kisung Ko ${ }^{5}$, Jeong Hwan Lee ${ }^{5}$, Jungmook Lyu ${ }^{3,6}$, Moon Hyang Park ${ }^{7}$, Jaeku \\ Kang $^{2,8}$, Jae Chan Kim ${ }^{\text {Corresp. } 4}$ \\ ${ }^{1}$ Departement of Ophthalmology, Konyang University Hospital, Daejeon, South Korea \\ 2 Myunggok Medical Research Institute, Konyang University, Daejeon, South Korea \\ 3 Myunggok Eye Research Institute, Konyang University, Daejeon, South Korea \\ 4 Departement of Ophthalmology, Chung-Ang University Hospital, Seoul, South Korea \\ 5 Therapeutic Protein Engineering Lab / College of Medicine, Chung-Ang University, Seoul, South Korea \\ 6 Department of Medical Science, Konyang University, Daejeon, South Korea \\ 7 Department of Pathology, Konyang University Hospital, Daejeon, South Korea \\ 8 Department of Pharmacology / College of Medicine, Konyang University, Daejeon, South Korea \\ Corresponding Author: Jae Chan Kim \\ Email address: jck50ey@daum.net
}

Background: Benzalkonium chloride (BAK), commonly used in glaucoma treatment, is an eye drop preservative with dose-dependent toxicity. Previous studies have observed the multi-functional benefits of angiogenin (ANG) against glaucoma. In our study, we evaluated ANG's cytoprotective effect on the trabecular meshwork (TM) damage induced by BAK. Additionally, we developed a plant-derived ANG fusion protein and evaluated its effect on TM structure and function.

Methods: We synthesized plant-derived ANG (ANG-FCK) by fusing immunoglobulin G's Fc region and KDEL to conventional recombinant human ANG (Rh-ANG) purified from transgenic tobacco plants. We established a mouse model using BAK to look for degenerative changes in the TM, and to evaluate the protective effects of ANG-FcK and Rh-ANG. Intraocular pressure (IOP) was measured for 4 weeks and ultrastructural changes, deposition of fluorescent microbeads, type I and IV collagen, fibronectin, laminin, and $\alpha$-SMA expression were analyzed after the mice were euthanized.

Results: TM structural and functional degeneration were induced by $0.1 \%$ BAK instillation in mice. ANG co-treatment preserved TM outflow function, which we measured using IOP and a microbead tracer. ANG prevented phenotypic and ultrastructure changes, and that protective effect might be related to the antifibrosis mechanism. We observed a similar cytoprotective effect in the BAK-induced degenerative TM mouse model, suggesting that plant-derived ANG-FCK could be a promising glaucoma treatment. 


\section{Plant-derived angiogenin fusion protein's}

\section{3 cytoprotective effect on trabecular meshwork damage}

\section{4 induced by Benzalkonium chloride in mice}

8 Jae Hoon Jeong ${ }^{1,2,3}$, Soo Jin Lee ${ }^{4}$, Kisung $\mathrm{Ko}^{5}$, Jeong Hwan Lee ${ }^{5}$, Jungmook Lyu ${ }^{3,6}$, Moon

9 Hyang Park ${ }^{7}$, Jaeku Kang ${ }^{2,8}$, Jae Chan $\mathrm{Kim}^{4}$

${ }^{1}$ Departement of Ophthalmology, Konyang University Hospital, Daejeon, Republic of Korea

${ }^{2}$ Konyang University Myunggok Medical Research Institute, Daejeon, Republic of Korea

${ }^{3}$ Konyang University Myunggok Eye Research Institute, Daejeon, Republic of Korea

${ }^{4}$ Department of Ophthalmology, Chung-Ang University Hospital, Seoul, Republic of Korea

${ }^{5}$ Therapeutic Protein Engineering Lab, College of Medicine, Chung-Ang University, Seoul,

Republic of Korea

${ }^{6}$ Department of Medical Science, Konyang University, Daejeon, Republic of Korea

${ }^{7}$ Department of Pathology, Konyang University Hospital, Daejeon, Republic of Korea

${ }^{8}$ Department of Pharmacology, College of Medicine, Konyang University, Daejeon, Republic of

Korea 
24 Corresponding Author

25 Jae Chan Kim, MD, PhD

26 Department of Ophthalmology, Chung-Ang University Hospital, Seoul, Republic of Korea

27 E-mail: jck50ey@daum.net 


\section{ABSTRACT}

Background: Benzalkonium chloride (BAK), commonly used in glaucoma treatment, is an eye drop preservative with dose-dependent toxicity. Previous studies have observed the multifunctional benefits of angiogenin (ANG) against glaucoma. In our study, we evaluated ANG's cytoprotective effect on the trabecular meshwork (TM) damage induced by BAK. Additionally, we developed a plant-derived ANG fusion protein and evaluated its effect on TM structure and function.

Methods: We synthesized plant-derived ANG (ANG-FcK) by fusing immunoglobulin G's Fc region and KDEL to conventional recombinant human ANG (Rh-ANG) purified from transgenic tobacco plants. We established a mouse model using BAK to look for degenerative changes in the TM, and to evaluate the protective effects of ANG-FcK and Rh-ANG. Intraocular pressure (IOP) was measured for 4 weeks and ultrastructural changes, deposition of fluorescent microbeads, type I and IV collagen, fibronectin, laminin, and $\alpha$-SMA expression were analyzed after the mice were euthanized.

Results: TM structural and functional degeneration were induced by $0.1 \% \mathrm{BAK}$ instillation in mice. ANG co-treatment preserved TM outflow function, which we measured using IOP and a microbead tracer. ANG prevented phenotypic and ultrastructure changes, and that protective effect might be related to the anti-fibrosis mechanism. We observed a similar cytoprotective effect in the BAK-induced degenerative TM mouse model, suggesting that plant-derived ANGFcK could be a promising glaucoma treatment. 


\section{Introduction}

Glaucoma is a progressive optic neuropathy associated with various risk factors, including increased intraocular pressure (IOP) (Van Buskirk \& Cioffi, 1992). IOP-related aqueous humor dynamics are currently the only known controllable factors for disease progression prevention. IOP-lowering eye drops contain therapeutic agents and additives (Inoue, 2014) such as benzalkonium chloride (BAK), a common ophthalmic preservative agent. Preservatives used in topical eye drops may cause ocular surface disorders, including superficial punctate keratitis, corneal erosion, conjunctival allergy, conjunctival injection, and anterior chamber inflammation (Baudouin, 2008; Noecker \& Miller, 2011; Rosin \& Bell, 2013).

After repeated instillations, BAK penetrates healthy eyes and is detected in both ocular surface structures and deeper tissues, such as the trabecular meshwork (TM) and optic nerve (BrignoleBaudouin et al., 2012). Eye drop preservatives may cause long-term trabecular degeneration and increased outflow resistance (Baudouin et al., 2012; Chang et al., 2015). Chronic or repeated eye drop use can have dose-dependent toxic effects, and should be examined when managing glaucoma. Although there are currently many anti-glaucomatous drugs formulated without preservatives, BAK is still frequently used in medicated eyedrops. Therefore, investigating protective agents against BAK-induced TM damage may improve the treatment and prevention of glaucoma.

Angiogenin (ANG), also known as Ribonuclease 5, has various functions (Gao \& Xu, 2008) and associations with cancer and neurological diseases via its roles in angiogenesis and apoptosis suppression (Li et al., 2012; Steidinger et al., 2011; Tello-Montoliu et al., 2006). ANG is highly concentrated in normal tear fluid that has pooled overnight and helps maintain corneal avascularity. It is suggesting that ANG plays a physiological role which is separate from its 
78 angiogenic role under normal ocular surface conditions (Sack et al., 2005). In addition, it has

79 been reported that ANG could be a candidate survival booster for transformed human TM cell

80 lines (Kim et al., 2016). However, the complete effects of BAK at the TM ultrastructure level

81 and ANG's protective mechanism are unclear. Since the trabecular outflow pathway of mice is

82 structurally and functionally similar to that of primates (Overby et al., 2014), we examined the

83 protective effect of ANG against BAK in mice TM. Additionally, considering that recombinant

84 human ANG (Rh-ANG) is expensive and plants are beneficial as a heterologous expression

85 system for large scale recombinant protein production (Jamal et al., 2009), we developed a plant-

86 derived ANG fusion protein using molecular farming. The aim of our study was to evaluate

87 ANG's efficacy in protecting TM structure and function, and to introduce molecular farming

88 technology to the ophthalmology field. 
90

91

92

93

94

\section{Materials and Methods}

\section{BAK-induced TM degenerative mouse model}

All mouse experiments were conducted in compliance with the Association for Research in Vision and Ophthalmology Statement for the Use of Animals in Ophthalmic and Vision Research. The Institutional Animal Care and Use Committee in College of Medicine, Konyang University reviewed and approved the study protocol (P-16-22-A-01). The following toxicity model was used: instillation of one drop of $0.01 \%, 0.02 \%, 0.1 \%$, and $0.2 \%$ BAK (Sigma Aldrich, Fluka, Buchs, Switzerland), respectively, twice a day (at 8 am and $8 \mathrm{pm}$ ) for 1 month, and a subconjunctival injection of $10 \mu \mathrm{L}$ of $0.1 \%$ BAK. The mice's contralateral eye served as shamoperated controls. Subconjunctival intramuscular injection of a tiletamine and zolazepam-mixed agent (2 mg/kg, Zoletil; Virbac, Fort Worth, TX, USA) and xylazine (6 mg/kg, Rompun; Bayer, Leverkusen, Germany) was performed under general anesthesia.

Following a previous study where six rats were used to model BAK-induced TM degeneration (Baudouin et al., 2012), we initially assigned five mice to each toxicity model. However, four to five mice were included in each group due to unexpected deaths during the follow-up period. They were all male C57BL/6J Jms SLC mice (7 weeks old; 21-24 g) purchased from SLC Laboratory (Hamamatsu, Shizuoka, Japan). The mice were housed in clear cages with 12-hour light/12-hour dark cycles at $30-70 \%$ humidity and $22-24^{\circ} \mathrm{C}$. Before BAK administration, the mice were allowed to acclimatize for 1 week and were provided tap water and food ad libitum. Their IOP was measured at 6 pm daily using a rebound tonometer (Tono-lab, iCare, Vantaa, Finland) without sedation, and we recorded the average values of three consecutive measurements. After 16 weeks, the mice were euthanized, and their eyes were enucleated for 
112 histological analyses. The experimental protocols are summarized in Fig S1.

113

114 Plant-derived ANG-FcK protein development

115 We cloned cDNA fragments encoding the human ANG fused Fc region of immunoglobulin G-

116 tagged endoplasmic reticulum retention signal, KDEL (ANG-FcK), into a pBI121 plant

117 expression vector. The gene was then inserted with the alfalfa mosaic virus untranslated leader

118 sequence (AMV) from RNA4 under the control of the cauliflower mosaic virus 35S promoter

119 into the vector. We transferred the ANG-FcK gene expression cassette as a HindIII-EcoRI

120 fragment into the plant binary vector pBI121, and conducted Agrobacterium-mediated plant

121 transformation using the vector to generate transgenic tobacco (Nicotiana tabacum) lines

122 expressing ANG-FcK.

123 We homogenized $100 \mathrm{mg}$ of transgenic plant leaf tissue in $300 \mu \mathrm{L}$ of $1 \times \mathrm{PBS}$, resolved the

124 plant extracts by $12.5 \%$ sodium dodecyl sulfate polyacrylamide gel electrophoresis (SDS-

125 PAGE), and transferred them to a nitrocellulose membrane (Millipore, Bedford, MA, USA). The

126 membrane was incubated in blocking solution $[5 \%(\mathrm{w} / \mathrm{v})$ skim milk (Fluka) in $1 \times \mathrm{TBS}, 0.05 \%$

127 (v/v) Tween 20 (TBST)], followed by a primary anti-ANG antibody (1:250, Abcam Inc.,

128 Cambridge, MA, USA), and an anti-mouse IgG2a Fc fragment (1:3000, Jackson

129 ImmunoResearch Labs, West Grove, PA) conjugated to horseradish peroxidase was used as the

130 secondary antibody to detect ANG-FcK. The anti-ANG antibody we used for immunoblotting

131 recognized the full length ANG protein (Cat.\# ab10600, Abcam Inc., Cambridge, MA, USA).

132 We used SuperSignal chemiluminescence substrate (Pierce, Rockford, IL, USA) to detect the

133 signal. Rh-ANG (R\&D Systems, Minneapolis, MN, USA) was used as a positive control.

134 We used the same method of purifying plant-derived ANG-FcK as in our previous paper (Lim 
135 et al., 2014). To purify plant-derived ANG-FcK, tobacco leaves were mixed with cold extraction 136 buffer (37.5 mM Tris- $\mathrm{HCl}$ pH 7.5, $50 \mathrm{mM} \mathrm{NaCl}, 15 \mathrm{mM}$ EDTA, 75 mM sodium citrate, and

$1370.2 \%$ sodium thiosulfate) and were homogenized in a HR2094 blender (Philips, Seoul, Korea).

138 After homogenization, the leaves were centrifuged for 30 minutes at $8800 \times \mathrm{g}$ at $4{ }^{\circ} \mathrm{C}$, the

139 supernatant was filtered using Miracloth (Merck, Darmstadt, Germany), and extra pure acetic

140 acid was added to adjust the $\mathrm{pH}$ to 5.1 . We centrifuged the solution at $10200 \times g$ for $30 \mathrm{~min}$ at

$1414^{\circ} \mathrm{C}$, brought up the $\mathrm{pH}$ to 7.0 by adding $3 \mathrm{M}$ Tris- $\mathrm{HCl}$, and added ammonium sulfate to a

142 saturation of $8 \%$. After centrifugation at $8800 \times g$ for $30 \mathrm{~min}$ at $4{ }^{\circ} \mathrm{C}$, we discarded the precipitate

143 and added ammonium sulfate to the supernatant to $40 \%$ saturation. After overnight incubation at

$1444^{\circ} \mathrm{C}$, the solution was centrifuged, the pellet was resuspended in extraction buffer to $1 / 10$ of the

145 original volume, and the final solution was centrifuged at $10200 \times g$ for $30 \mathrm{~min}$ at $4^{\circ} \mathrm{C}$. The

146 supernatant was filtered through a $0.45-\mathrm{mm}$ filter and loaded onto a HiTrap Protein A column

147 (Pharmacia, Uppsala, Sweden). We applied soluble protein extract to a protein A column (GE

148 Healthcare, Piscataway, NJ, USA) and dialyzed elutes of plant-derived ANG-FcK protein against

$1491 \times$ PBS buffer. Aliquots were frozen in liquid nitrogen and stored at $-80^{\circ} \mathrm{C}$ for glycosylation

150 analysis.

151

152 ANG treatment on the experimental mouse model

153 We used the $0.1 \%$ BAK treatment toxicity model twice daily for 1 month to maximize the 154 toxic effect. Two types of ANG (Rh-ANG and ANG-FcK) were used, and $4 \mu \mathrm{L}$ of ANG (50 $155 \mu \mathrm{g} / \mathrm{mL}$ ) was administered to mice twice daily for 1 month. We arranged the combinations of 156 toxic and protective substances into six groups: BAK, Rh-ANG, ANG-FcK, Rh-ANG with BAK,

157 ANG-FcK with BAK, and sham-treated control. In each experimental group, mice were analyzed 
158 using three different methods: three underwent ultrastructural analysis, three underwent

159 immunohistochemical analysis, and three underwent microbead injection to analyze the outflow

160 pathway. Mice were treated with ANG 3 days before BAK administration and the two

161 substances were administered at 10-minute intervals.

162 IOP was measured at $6 \mathrm{pm}$ daily without sedation, and mice were euthanized 4 weeks after

163 BAK and/or ANG treatment. Their eyes were then prepared for electron microscopy or

164 immunohistochemistry. Microbeads were injected into three eyes in each experimental group

165 before mice were sacrificed under general anesthesia to evaluate the conventional outflow

166 pathway. The anterior chambers of eyes were cannulated with a 30-gauge needle connected by

167 tubing to a $1-\mathrm{mL}$ syringe filled with green fluorescent beads $(100 \mathrm{~nm}$, carboxylate modified

168 FluoSpheres, 1:750 dilution; Molecular Probes, Eugene, OR, USA) and were loaded into a

169 microdialysis infusion pump (World Precision Instruments, Sarasota, FL, USA). $10 \mu \mathrm{L}$ of liquid

170 was infused into the anterior chamber at $0.167 \mu \mathrm{L} / \mathrm{min}$ for 1 hour. The experimental protocols are

171 summarized in Fig. S1.

\section{Immunohistochemical and ultrastructural analyses}

173 We embedded and froze 36 eyes in Optimal Cutting Temperature Compound (Tissue-Tek, Cat \#4583; Sakura Americas, Torrance, CA, USA). Sagittal cryosectioning was performed through the entire anterior-posterior extension of the globe at a thickness of $10-\mu \mathrm{m}$. Sections were stored at $-80^{\circ} \mathrm{C}$ and dried for 10 minutes at room temperature. After we washed the sections three times with PBS (Welgene, Gyeongsangbuk-do, Korea) for 10 minutes each, we drew circles along the tissues using a PAP pen (Vector, Burlingame, CA, USA). The sections were fixed with 4\% paraformaldehyde (Santa Cruz Biotechnology, Santa Cruz, CA, USA) for 15 minutes, incubated with $0.1 \%$ Triton X-100 (Sigma-Aldrich, St. Louis, MO, USA) for 5 minutes for 
181 permeabilization, and washed three times with PBS for 10 minutes each. Slides were incubated 182 in PBS and 1\% BSA (Gibco; Thermo Fisher Scientific, Inc.) for 1 hour at room temperature for 183 blocking. Sections were washed once for 10 minutes and incubated with primary antibodies in 184 blocking solution at $4^{\circ} \mathrm{C}$ overnight. Primary antibodies included collagen type I (ab34710, 185 1:100; Abcam), collagen type IV (ab6586, 1:100; Abcam), fibronectin (sc-69681, 1:100; Santa 186 Cruz), laminin (ab11575, 1:100; Abcam), and $\alpha$-smooth muscle actin ( $\alpha$-SMA) (sc-53142, 1:100; 187 Santa Cruz). After being washed three times (10 minutes each), sections were incubated for 1 188 hour with Cy2 (green) and Cy3 (red) secondary antibodies (1:250; Jackson ImmunoResearch, 189 West Grove, PA, USA). Sections were washed, counter-stained with Hoechst 33258 (1:1000), 190 and mounted with a drop of AquaPolyMount (Polysciences, Warrington, PA, USA). We obtained images using the fluorescence microscope, Imager D2 (Zeiss, Oberkochen, Germany).

192 For ultramicroscopy, we fixed 18 eyes overnight in cold $2.5 \%$ glutaraldehyde, then in 1 osmium tetroxide for 1 hour. After dehydration in a graded acetone series, tissues were embedded in Epon resin, and $0.5-\mu \mathrm{m}$ semithin or 70-nm ultrathin sections were cut using an ultramicrotome. Semithin sections were stained with toluidine blue. Ultrathin sections were placed on 200-mesh copper grids and double stained with 4\% uranyl acetate for 20 minutes and $0.2 \%$ lead citrate for 5 minutes. To obtain tangential sections parallel to the plane of the inner wall, we took consecutive semithin sections through the cornea and sclera in a plane parallel to the limbus's outer surface. Once we reached the lumen of Schlemm's canal (SC), we removed consecutive ultrathin sections until we reached the inner wall of SC, juxtacanalicular connective tissue (JCT), and the lamellated TM. Semithin sections were viewed using an Olympus CX22 microscope (Tokyo, Japan), and ultrathin sections were viewed using an electron microscope (HT7700; Hitachi High-Tech Science Corp., Tokyo, Japan) at 80 kV. 
205 Statistical analysis

206 Our results are expressed as means \pm standard errors, and normality and equal variances in 207 groups were tested. Analysis of variance (ANOVA) was used to analyze IOP differences across 208 three or more groups at each timepoint, and repeated measures ANOVA was used to compare the 209 baseline IOP during the follow-up period. We included Tukey's tests, Bonferroni's methods, 210 Duncan's tests, and Dunnett's T3 tests in post-hoc analyses. The probability level for statistical 211 significance was set at 5\%. Data were recorded and analyzed using SPSS for Windows, version 21218.0 (SPSS Inc., Chicago, IL, USA). 


\section{Results}

214

\section{BAK effect on intraocular pressure}

216 The mean IOP changes in response to various BAK concentrations are shown in Fig. 1A.

217 After 2 weeks of treatment twice a day, $0.1 \%$ and $0.2 \%$ BAK increased the mean IOP. The IOP

218 was higher in these groups than in the sham-operated control group over the 16-week period, 219 although treatment was performed for 4 weeks. At 4 weeks, $0.1 \%$ BAK treatment significantly 220 induced an increase in IOP by approximately $36 \%(17.3 \pm 1.0 \mathrm{mmHg})$ compared to that of the 221 control group $(12.7 \pm 0.6 \mathrm{mmHg}, P<0.01)$. This group's IOP remained higher at 6 weeks $(23.8$

$222 \pm 1.2 \mathrm{mmHg})$ and 12 weeks $(24.6 \pm 2.3 \mathrm{mmHg})$ than the control group at the same points in time $223(14.8 \pm 3.0 \mathrm{mmHg}$ and $16.1 \pm 5.1 \mathrm{mmHg}, P<0.01)$.

224 The mean IOP was significantly higher in the $0.2 \%$ BAK group than in the control group at 4 225 weeks $(16.5 \pm 2.4 \mathrm{mmHg}, P=0.02)$ and at 6 weeks $(21.1 \pm 1.3 \mathrm{mmHg}, P<0.01)$. The IOP under $0.01 \%$ and $0.02 \%$ BAK treatments and subconjunctival $0.1 \%$ BAK injection was not significantly different from that of the control group, except for $0.02 \%$ BAK at 1 week $(10.3 \pm$ $1.3 \mathrm{mmHg}$ vs. $14.9 \pm 1.6 \mathrm{mmHg}$ in the control group; $P=0.03)$. In the toxic BAK-induced TM degeneration group, we administered a $0.1 \%$ BAK treatment twice a day for 4 weeks.

\section{Expression and purification of ANG-FcK in transgenic plants}

232 We examined ANG-FcK expression in randomly selected transgenic plants using western blotting (Fig. 2A). The Rh-ANG protein band was detected at approximately $15 \mathrm{kDa}$ and ANGFcK was detected at approximately $44 \mathrm{kDa}$. No band was observed in the non-transgenic plant.

235 We purified ANG-FcK from leaves harvested from transgenic tobacco plants. Protein A column 
236 purification yielded an average of $2 \mathrm{mg}$ of plant-derived ANG-FcK per $\mathrm{kg}$ of fresh leaves from a

237 line with high protein expression. SDS-PAGE analysis of purified ANG-FcK revealed one major 238 band (44 kDa, Fig. 2B).

239

240

Effect of ANG on BAK-induced changes in intraocular pressure

241 The mean IOP after $0.1 \%$ BAK treatment continued to increase and was significantly higher 242 than that of the other groups at 3 weeks $(15.2 \pm 2.1 \mathrm{mmHg}, P<0.01)$ and 4 weeks $(15.7 \pm 1.7$

$243 \mathrm{mmHg}, P<0.01$ ) (Fig. 2C and 2D). There were few IOP differences between the single Rh244 ANG or ANG-FcK treatments and the control group, and inter- and intra-group variability was 245 low (Fig. 2E). For treatments with Rh-ANG or ANG-FcK with BAK, the mean IOP was similar 246 to those of single Rh-ANG and ANG-FcK treatments and the control group at 3 weeks. However 247 at 4 weeks, Rh-ANG with BAK was elevated to $12.1 \pm 1.8 \mathrm{mmHg}(P<0.01)$ and ANG-FcK 248 with $\mathrm{BAK}$ to $11.6 \pm 0.4 \mathrm{mmHg}(P<0.05)$, although these values were lower for the single BAK $249 \operatorname{group}(P<0.01)$ (Fig. 2C, 2ED and 2E).

\section{Immunohistochemical analysis of the effects of ANG on BAK response}

252

253

We observed that type I collagen's Cy3 labeling in the outflow tissue along the iridocorneal angle was more pronounced in BAK-treated eyes than in the single ANG and control groups (Fig. 3A). The type I collagen labeling in the TM region adjacent to the corneal endothelium was patchy and thin in the control (Fig. 3A) and single Rh-ANG (Fig. 3B) and ANG-FcK (Fig. 3C) groups. However, we observed more intense and broader labeling in the single BAK (Fig. 
259 Type IV collagen and fibronectin labeling was more prominent in BAK-treated eyes (especially

260 those in the single BAK-treated group) than in the single ANG and control groups. The positive

261 laminin labeling was more noticeable in the single BAK-treated group (Fig. 4 D) than in the Rh-

262 ANG with BAK (Fig. 4E) and ANG-FcK with BAK (Fig. 4F) groups, but was barely detectable

263 in the single ANG and control groups (Fig. 4A to 4C). We only detected spotty positive $\alpha$-SMA

264 labeling in the inner sclera of the TM layers adjacent to SC in the single BAK-treated group (Fig.

$2654 \mathrm{~J}$ ), not in any ANG-treated and control groups. In the ciliary body, type I, IV collagen, and

266 fibronectin labeling was observed in the epithelium, and laminin and $\alpha$-SMA labeling was more

267 apparent in the stroma layer (Fig. 3 and 4).

268 Fluorescent bead deposition traces the flow of aqueous humor, and its intensity is correlated 269 with TM outflow function (Li et al., 2016; Swaminathan et al., 2013). Green fluorescent beads

270 were present in all of the experimental groups' outflow tissues, but deposition intensity

271 decreased in the single BAK-treated group (Fig. 4P). The sparsely deposited fluorescent beads

272 suggest an abnormal TM outflow function resulting from BAK treatment. The cumulative bead

273 distribution data from the single BAK-treated group can be found in Fig. S2. The Rh-ANG and

274 ANG-FcK with BAK groups exhibited more prominent deposition of green fluorescent beads in

275 their outflow tissues, but these results were based on a qualitative analysis.

276

277

\section{TM histological and ultrastructural changes}

278 After BAK treatment, the characteristic structure of outflow tissues showed little difference under a light microscope (Fig. 1B to 1E). Based on our observations of the ultrathin sections, BAK treatment led to a thickening of the lamina beam in the TM. In particular, we observed 
282 SC's inner wall. These were prominent when compared with the empty spaces within the control 283 group's JCT (Fig. 5A and 5B). An increased density and multidirectional array of fibrillar 284 material were also seen in the single BAK-treated group (Fig. 5C and 5D), similar to the 285 "fingerprint"-like basement membrane described in human eyes treated with corticosteroids 286 (Johnson et al. 1997).

287 There were no remarkable differences between the TM ultrastructures of the Rh-ANG with 288 BAK group (Fig. 5E and 5F) and the ANG-FcK with BAK group (Fig. 5G and $5 \mathrm{H}$ ). These 289 groups showed some similarities such as a thickness of lamina beam and empty spaces within the 290 JCT. The fibrillar material was denser than that of the control group, but sparser than that of the 291 single BAK-treated group. Although we did not do a quantitative analysis, we found more 292 intracellular organelles in the ANG and BAK-treated groups than in the control group. 


\section{Discussion}

295 In this study, we examined the toxicity of chronic BAK exposure on the TM and ANG's

296

297

298

299

300

301

302

303

304

305

306

307

308

309

310

311

312

313

314

315

316

defenses against changes in the trabecular outflow pathway. We induced the structural and functional degeneration of the TM through BAK treatment in a mouse model. Co-treatment with ANG successfully preserved the outflow function of the TM, suggesting that ANG prevents fibrosis.

Additionally, we developed ANG-FcK, which has important practical applications. The greater molecular weight of ANG-FcK (44 kDa) over Rh-ANG (15 kDa) enhanced protein stability, facilitated purification, and improved yield. ANG-FcK was similar to Rh-ANG with respect to IOP, flow of aqueous humor, and ultrastructural changes in the BAK-induced TM degenerative mouse model. Purifying plant-derived ANG-FcK yielded an average of $2 \mathrm{mg}$ per $\mathrm{kg}$ of fresh leaves. With a cost of approximately U.S. 1,000 dollars per $250 \mu \mathrm{g}$ of Rh-ANG, $1 \mathrm{~kg}$ of transgenic plants is worth approximately U.S. 8,000 dollars of conventional protein. To the best of our knowledge, this is the first study to apply molecular farming techniques in ophthalmology, and our production of recombinant ANG may be beneficial to this field.

Although BAK is the most common preservative used in ophthalmic solutions, its effects on IOP or outflow in vivo have not been explored (Rasmussen et al. 2014). After $0.1 \%$ and $0.2 \%$ BAK topical drops delivered twice a day for 4 weeks in our mouse model, the IOP rose significantly at 4 weeks and remained elevated for 2 additional weeks. This was higher than the IOP for other concentrations and the $0.1 \%$ BAK subconjunctival injection. These findings differ from the results of Baudouin et al. (2012) who found that IOP significantly increased 7 days after $100 \mu \mathrm{L}$ of $0.01 \%$ BAK subconjunctival injection and remained high for 6 additional days after a second injection on day 7. A major reason for these differences could be the animal model used. 
317 Baudouin et al. (2012) injected $100 \mu \mathrm{L}$ of BAK into the subconjunctival space of rats weighing

318300 to $350 \mathrm{~g}$. In our study, we performed a single injection of $10 \mu \mathrm{L}$ into the subconjunctival

319 space of mice weighing 21 to $24 \mathrm{~g}$. This might explain the lack of a significant change in IOP

320 despite a higher BAK concentration. In a mouse model, it is difficult to inject $10 \mu \mathrm{L}$ into the

321 subconjunctival space without any losses. Moreover, the administration of topical drops is more

322 suitable for a chronic exposure model.

323 The IOP of the combination of ANG and BAK was lower than that the IOP of BAK alone, but

324 was greater than that of eyes not exposed to BAK at 4 weeks. Our results are in agreement with

325 the findings of an earlier experimental study (Kim et al., 2016) where ANG lowered IOP in

326 both normal and elevated rat models using the vortex vein cauterization method. ANG also

327 conserved the conventional outflow of aqueous humor via the TM after BAK treatment.

328 Fluorescent beads were deposited along the outflow tract in the ANG and control groups, but

329 were sparse in the BAK-induced toxicity model. The cumulative distribution of microbeads was

330 sparse across the parallel sections of all three single BAK-treated mouse models (Fig. S2).

331 Although it was based on a subjective analysis since there are low and high-flow regions in the

332 360-degree circumference of the TM, these results indicate that BAK creates an abnormal

333 outflow of aqueous humor, which is consistent with previous results (Swaminathan et al., 2013;

334 Zhang et al., 2009). Cross-sectional images cannot represent the whole TM, but analyses of

335 fluorescence intensities on flat-mounted sections have been suggested as helpful for this task.

336 Both the lamina thickness and fibrillar material density increased, and we observed type I,

337 type IV collagen, fibronectin, laminin, and $\alpha$-SMA fibrogenic markers (Faralli et al., 2019; Ko \&

338 Tan, 2013; Pattabiraman et al., 2014) in the BAK-induced toxicity model. BAK caused an

339 epithelial mesenchymal transition-like phenomenon and myofibroblast-like phenotypic changes 
340 in the TM. These changes caused TM cells to abundantly express fibronectin, activate motility,

341 and switch to a myofibroblast-like phenotype, simultaneously strengthening the actin

342 cytoskeleton and extracellular matrix. Fibronectin regulates the deposition of collagen IV and

343 laminin (Faralli et al., 2019). Overall, these changes cause an increase in TM resistance to

344 aqueous humor outflow (Takahashi et al., 2014; Tamm, 2013). In eyes co-treated with ANG and

345 BAK, we did not detect $\alpha$-SMA, and the overall ultrastructural configuration was similar to that

346 of the control. However, the fibrillar material density had increased and extracellular matrix

347 markers such as collagen IV, fibronectin, and laminin were more abundant than in the single

348 ANG and control groups. These findings confirm that ANG defends against fibrosis and

349 myofibroblast-like phenotypic changes induced by BAK by maintaining the proper ultrastructure

350 for aqueous humor outflow.

351 Our study has its limitations. First, the BAK concentration we used was higher than those used

352 in commercial eye drops. However, we used BAK that had accumulated in the TM, iris, and lens

353 samples during cataract and glaucoma surgery in patients after long-term administration of BAK-

354 containing medication (Desbenoit et al., 2013). Second, the immunohistochemical and

355 ultrastructural findings in our study were based on subjective analyses. Quantifiable methods

356 such as measuring effective filtration areas on anterior segment images (Li et al., 2016;

357 Swaminathan et al., 2013) and ultrastructural analysis of basement membrane material length

358 (Overby et al., 2014) are needed for more comprehensive data. Third, extra in vitro experimental

359 studies are necessary to investigate the protective mechanism of ANG against BAK. Previous

360 studies showed that ANG may activate Akt-mediated signals for nitric oxide production and TM

361 remodeling by regulating matrix metalloproteinase and rho-kinase (Kim et al., 2016). Finally,

362 further research on ANG's effect on retinal ganglion cells may clarify its function and improve 
363 its clinical effectiveness. Because glaucoma is an ocular neurodegenerative disease characterized

364 by the progressive death of retinal ganglion cells, the importance of ANG enrichment in normal

365 motor neurons has been observed in studies on amyotrophic lateral sclerosis, a fetal

366 neurodegenerative disease (Kieran et al., 2008).

367

\section{Conclusions}

369 In conclusion, ANG's protective effect on TM may involve an anti-fibrotic function with a

370 less extensive ultrastructural change that retains outflow function than exposure to single toxic

371 substance such as BAK. Plant-derived ANG-FcK's protective effect is similar to that of Rh-

372 ANG, and it is a promising candidate for an alternative eye drop additive. Future studies should

373 focus on ANG's detailed defense mechanism and potential applications in glaucoma

374 management.

375

376 Acknowledgements

377 The authors wish to thank medical laboratory technologist Dae Young Kim, Department of

378 Pathology, Konyang University Hospital, Daejeon, Korea, for their electron microscopy

379 technical support. 
381

382

383

384

385

386

387

388

389

390

391

392

393

394

395

396

397

398

399

400

401

402

403

\section{References}

Baudouin C. 2008. Detrimental effect of preservatives in eyedrops: implications for the treatment of glaucoma. Acta Ophthalmol 86:716-726. 10.1111/j.1755-3768.2008.01250.x

Baudouin C, Denoyer A, Desbenoit N, Hamm G, and Grise A. 2012. In vitro and in vivo experimental studies on trabecular meshwork degeneration induced by benzalkonium chloride (an American Ophthalmological Society thesis). Trans Am Ophthalmol Soc 110:40-63.

Brignole-Baudouin F, Desbenoit N, Hamm G, Liang H, Both JP, Brunelle A, Fournier I, Guerineau V, Legouffe R, Stauber J, Touboul D, Wisztorski M, Salzet M, Laprevote O, and Baudouin C. 2012. A new safety concern for glaucoma treatment demonstrated by mass spectrometry imaging of benzalkonium chloride distribution in the eye, an experimental study in rabbits. PLoS One 7:e50180. 10.1371/journal.pone.0050180

Chang C, Zhang AQ, Kagan DB, Liu H, and Hutnik CM. 2015. Mechanisms of benzalkonium chloride toxicity in a human trabecular meshwork cell line and the protective role of preservative-free tafluprost. Clin Exp Ophthalmol 43:164-172. 10.1111/ceo.12390

Desbenoit N, Schmitz-Afonso I, Baudouin C, Laprevote O, Touboul D, Brignole-Baudouin F, and Brunelle A. 2013. Localisation and quantification of benzalkonium chloride in eye tissue by TOF-SIMS imaging and liquid chromatography mass spectrometry. Anal Bioanal Chem 405:4039-4049. 10.1007/s00216-013-6811-7

Faralli JA, Filla MS, and Peters DM. 2019. Role of Fibronectin in Primary Open Angle Glaucoma. Cells 8. 10.3390/cells8121518

Gao X, and Xu Z. 2008. Mechanisms of action of angiogenin. Acta Biochim Biophys Sin (Shanghai) 40:619-624. 
404 Inoue K. 2014. Managing adverse effects of glaucoma medications. Clin Ophthalmol 8:903-913.

405

406

407

408

409

410

411

412

413

414

415

416

417

418

419

420

421

422

423

424

425

426

\subsection{7/OPTH.S44708}

Jamal A, Ko K, Kim HS, Choo YK, Joung H, and Ko K. 2009. Role of genetic factors and environmental conditions in recombinant protein production for molecular farming. Biotechnol Adv 27:914-923. 10.1016/j.biotechadv.2009.07.004

Johnson D, Gottanka J, Flugel C, Hoffmann F, Futa R, and Lutjen-Drecoll E. 1997. Ultrastructural changes in the trabecular meshwork of human eyes treated with corticosteroids. Arch Ophthalmol 115:375-383.

Kieran D, Sebastia J, Greenway MJ, King MA, Connaughton D, Concannon CG, Fenner B, Hardiman O, and Prehn JH. 2008. Control of motoneuron survival by angiogenin. $J$ Neurosci 28:14056-14061. 10.1523/JNEUROSCI.3399-08.2008

Kim KW, Park SH, Oh DH, Lee SH, Lim KS, Joo K, Chun YS, Chang SI, Min KM, and Kim JC. 2016. Ribonuclease 5 coordinates signals for the regulation of intraocular pressure and inhibits neural apoptosis as a novel multi-functional anti-glaucomatous strategy. Biochim Biophys Acta 1862:145-154. 10.1016/j.bbadis.2015.11.005

Ko MK, and Tan JC. 2013. Contractile markers distinguish structures of the mouse aqueous drainage tract. Mol Vis 19:2561-2570.

Li G, Mukherjee D, Navarro I, Ashpole NE, Sherwood JM, Chang J, Overby DR, Yuan F, Gonzalez P, Kopczynski CC, Farsiu S, and Stamer WD. 2016. Visualization of conventional outflow tissue responses to netarsudil in living mouse eyes. Eur $J$ Pharmacol 787:20-31. 10.1016/j.ejphar.2016.04.002

Li S, Yu W, and Hu GF. 2012. Angiogenin inhibits nuclear translocation of apoptosis inducing factor in a Bcl-2-dependent manner. J Cell Physiol 227:1639-1644. 10.1002/jcp.22881 
427 Lim CY, Lee KJ, Oh DB, and Ko K. 2014. Effect of the developmental stage and tissue position

428

429

430

431

432

433

434

435

436

437

438

439

440

441

442

443

444

445

446

447

448

449

on the expression and glycosylation of recombinant glycoprotein GA733-FcK in transgenic plants. Front Plant Sci 5:778. 10.3389/fpls.2014.00778

Noecker R, and Miller KV. 2011. Benzalkonium chloride in glaucoma medications. Ocul Surf 9:159-162.

Overby DR, Bertrand J, Tektas OY, Boussommier-Calleja A, Schicht M, Ethier CR, Woodward DF, Stamer WD, and Lutjen-Drecoll E. 2014. Ultrastructural changes associated with dexamethasone-induced ocular hypertension in mice. Invest Ophthalmol Vis Sci 55:49224933. 10.1167/iovs.14-14429

Pattabiraman PP, Maddala R, and Rao PV. 2014. Regulation of plasticity and fibrogenic activity of trabecular meshwork cells by Rho GTPase signaling. J Cell Physiol 229:927-942. 10.1002/jcp. 24524

Rasmussen CA, Kaufman PL, and Kiland JA. 2014. Benzalkonium chloride and glaucoma. $J$ Ocul Pharmacol Ther 30:163-169. 10.1089/jop.2013.0174

Rosin LM, and Bell NP. 2013. Preservative toxicity in glaucoma medication: clinical evaluation of benzalkonium chloride-free 0.5\% timolol eye drops. Clin Ophthalmol 7:2131-2135. 10.2147/OPTH.S41358

Sack RA, Conradi L, Krumholz D, Beaton A, Sathe S, and Morris C. 2005. Membrane array characterization of 80 chemokines, cytokines, and growth factors in open- and closed-eye tears: angiogenin and other defense system constituents. Invest Ophthalmol Vis Sci 46:1228-1238. 10.1167/iovs.04-0760

Steidinger TU, Standaert DG, and Yacoubian TA. 2011. A neuroprotective role for angiogenin in models of Parkinson's disease. J Neurochem 116:334-341. 10.1111/j.1471- 
450

451

452

453

454

455

456

457

458

459

460

461

462

463

464

465

466

467

468

469

470

471

472

4159.2010.07112.x

Swaminathan SS, Oh DJ, Kang MH, Ren R, Jin R, Gong H, and Rhee DJ. 2013. Secreted protein acidic and rich in cysteine (SPARC)-null mice exhibit more uniform outflow. Invest Ophthalmol Vis Sci 54:2035-2047. 10.1167/iovs.12-10950

Takahashi E, Inoue T, Fujimoto T, Kojima S, and Tanihara H. 2014. Epithelial mesenchymal transition-like phenomenon in trabecular meshwork cells. Exp Eye Res 118:72-79. 10.1016/j.exer.2013.11.014

Tamm ER. 2013. [Functional morphology of the outflow pathways of aqueous humor and their changes in open angle glaucoma]. Ophthalmologe 110:1026-1035. 10.1007/s00347-012$2670-4$

Tello-Montoliu A, Patel JV, and Lip GY. 2006. Angiogenin: a review of the pathophysiology and potential clinical applications. J Thromb Haemost 4:1864-1874. 10.1111/j.15387836.2006.01995.x

Van Buskirk EM, and Cioffi GA. 1992. Glaucomatous optic neuropathy. Am J Ophthalmol 113:447-452.

Zhang Y, Toris CB, Liu Y, Ye W, and Gong H. 2009. Morphological and hydrodynamic correlates in monkey eyes with laser induced glaucoma. Exp Eye Res 89:748-756. 10.1016/j.exer.2009.06.015 


\section{Figure 1}

Changes in intraocular pressure (IOP) in response to BAK in mouse models and representative histological stains of the mouse eye TM

(A) The mean IOP increased significantly in response to the instillation of $0.1 \%$ and $0.2 \%$ BAK at 4 weeks, and remained higher than that of the control group at 6 weeks, although the instillation was maintained for 4 weeks. Error bars represent standard errors of the mean. $* P$ $<0.05$ and ${ }^{* * P}<0.01$ (compared to the control group). (B) Hematoxylin and eosin (H-E) stain of the mouse eye TM under a light microscope. (C) H-E stain of the characteristic architecture of outflow tissues showed little difference by BAK. (D) Masson trichrome (M-T) stain of the mouse eye TM under a light microscope. (E) M-T stain of the characteristic architecture of outflow tissues showed little difference 16 weeks after BAK administration.
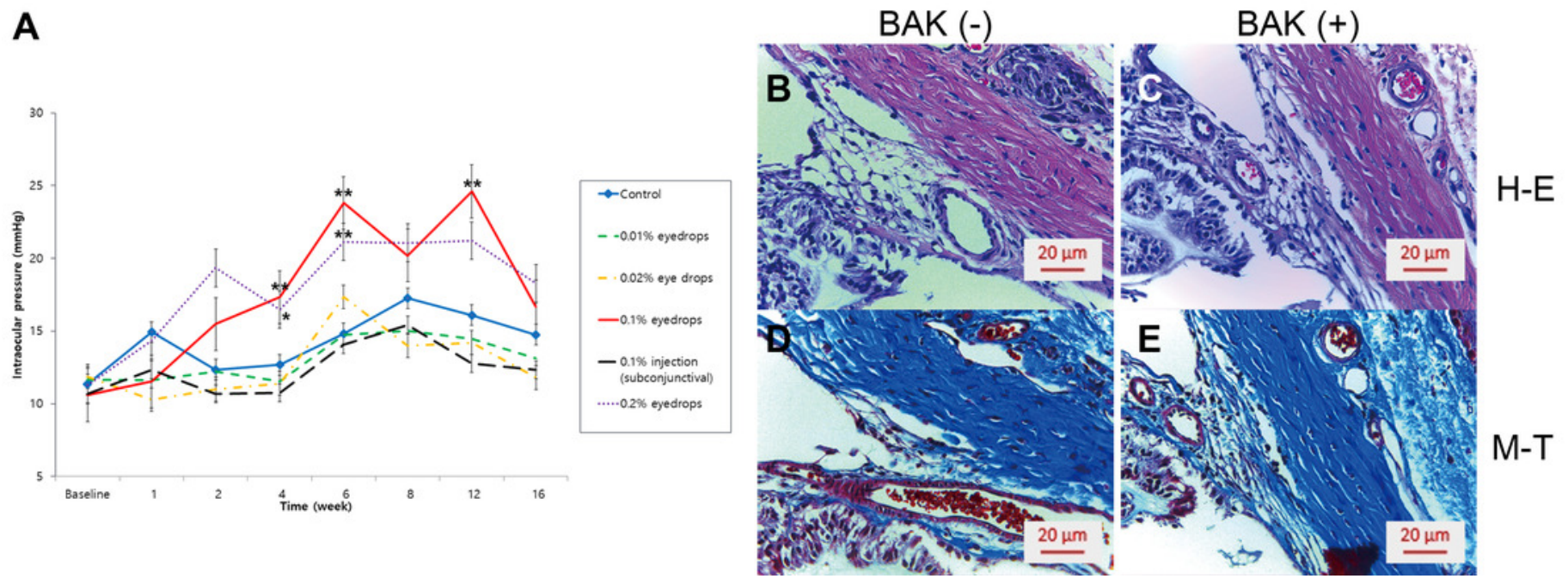


\section{Figure 2}

Development of ANG-FcK, and changes in the effects of BAK on intraocular pressure (IOP) in response to ANG

(A) Expression of ANG-FcK in randomly selected transgenic plants. (+), positive control, RhANG; (-), non-transgenic tobacco plant leaf extract. \#1167-1187, transgenic plant line number. (B) SDS-PAGE results for purified ANG-FcK. \#1-2, purified protein fraction number; Column through: plant extracts passed through a column. (C) The single BAK treatment induced the greatest elevation in mean IOP after 3 weeks among all groups. Cotreatment with Rh-ANG and BAK maintained the initial mean IOP over 3 weeks; however, IOP was elevated at 4 weeks, although it was lower than that for the single BAK treatment group. (D) The change in mean IOP for co-treatment with ANG-FCK and BAK was similar to that for RhANG. IOP was higher than that in the control group, but lower than that in the single BAK treatment group at 4 weeks. (E) There was no significant difference in mean IOP between RhANG and ANG-FcK for the combined use with BAK. Error bars represent standard errors of the mean. $* P<0.05$ and $* * P<0.01$ 
A

Leaf extracts
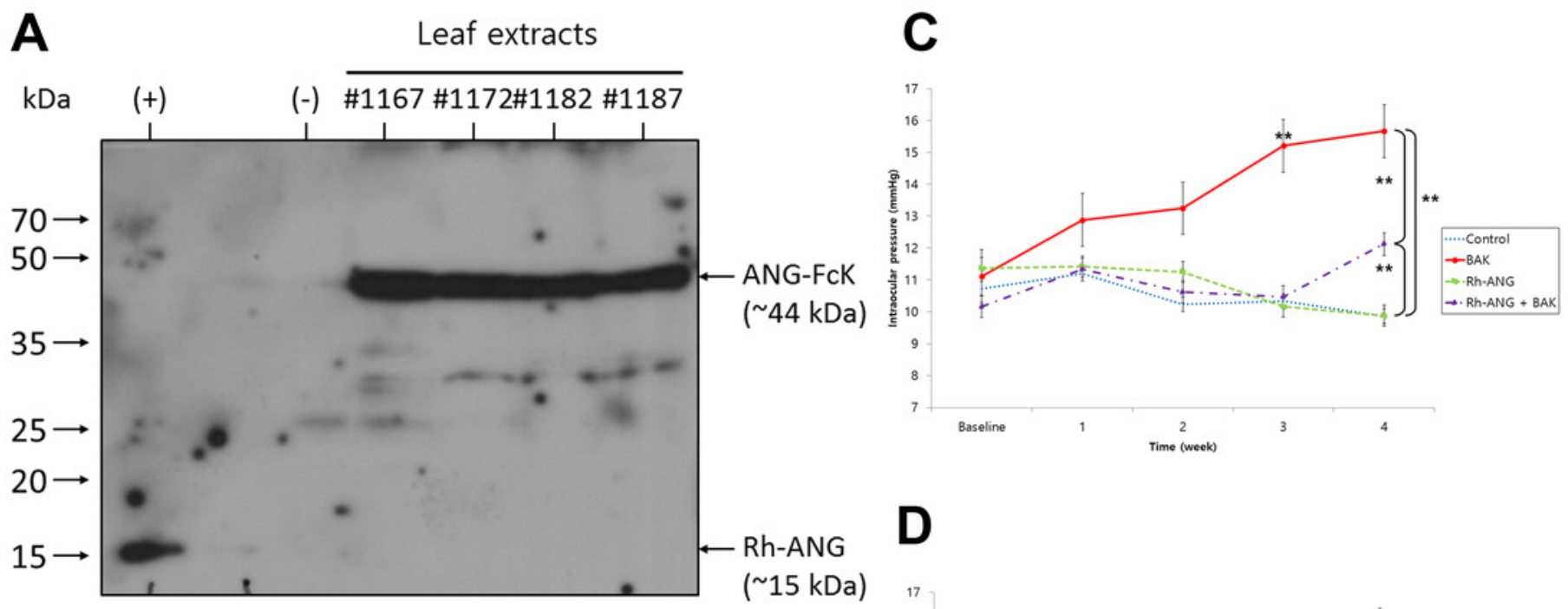

purified

ANG-FcK

$\begin{array}{lll}\text { B } & \text { fractions } & \text { Column } \\ & \text { Marker BSA } & \# 1 \quad \text { \#2 } \\ \text { through }\end{array}$

D
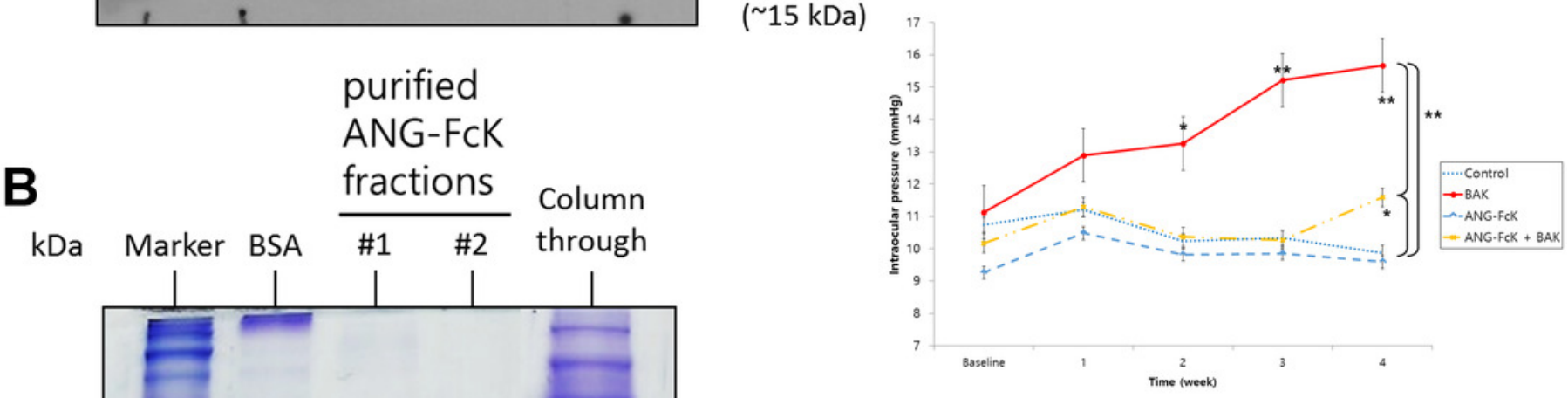

־ ANG-FcK ( $\sim 4 \mathrm{kDa})$

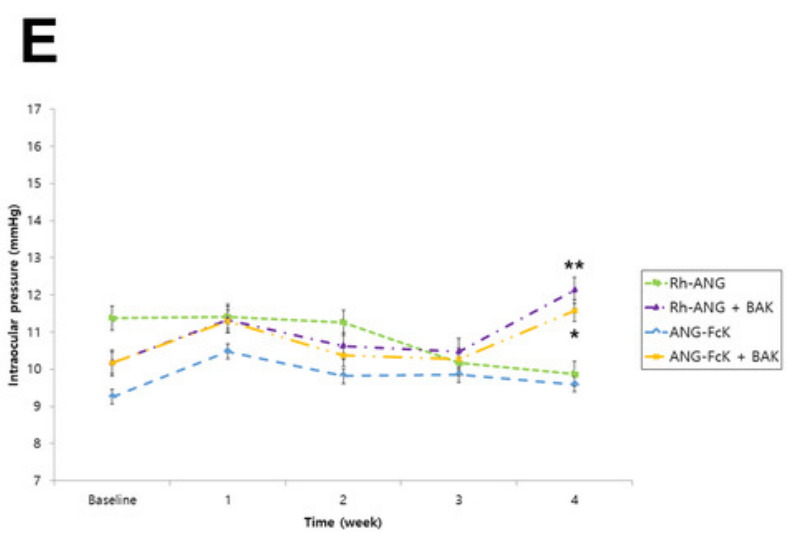




\section{Figure 3}

Immunohistochemical labeling of aqueous outflow tissues in a mouse model 01

(A) Sham-treated control. (B) Single Rh-ANG treatment. (C) Single ANG-FcK treatment. (D)

Single BAK treatment. (E) Rh-ANG with BAK treatment. (F) ANG-FcK with BAK treatment group. Staining type I collagen (red) was present in outflow tissues (dotted oval) of the TM and the inner and outer walls of Schlemm's canal in sham-treated control and ANG-treated mice, but the distribution tended to be patchy and thin. BAK-treated mice, in contrast, showed more intense and broader labeling in inner walls of Schlemm's canal (arrows). Nuclei were counter-stained with Hoechst (blue). (G) Sham-treated control. (H) Single Rh-ANG treatment. (I) Single ANG-FcK treatment. (J) Single BAK treatment. (K) Rh-ANG with BAK treatment. (L) ANG-FcK with BAK treatment group. Staining of type IV collagen (green) was more prominent in BAK-treated mice (arrows) and especially in eyes of the single BAKtreated group than in single ANG and sham-treated control groups. (M) Sham-treated control. (N) Single Rh-ANG treatment. (O) Single ANG-FcK treatment. (P) Single BAK treatment. (Q) Rh-ANG with BAK treatment. (R) ANG-FCK with BAK treatment group. Staining of Fibronectin (red) was more prominent in BAK-treated mice (arrows) and especially in eyes of the single BAK-treated group than in single ANG and sham-treated control groups. All images were magnified 200 times. Veh $=$ vehicle for sham-treated control 

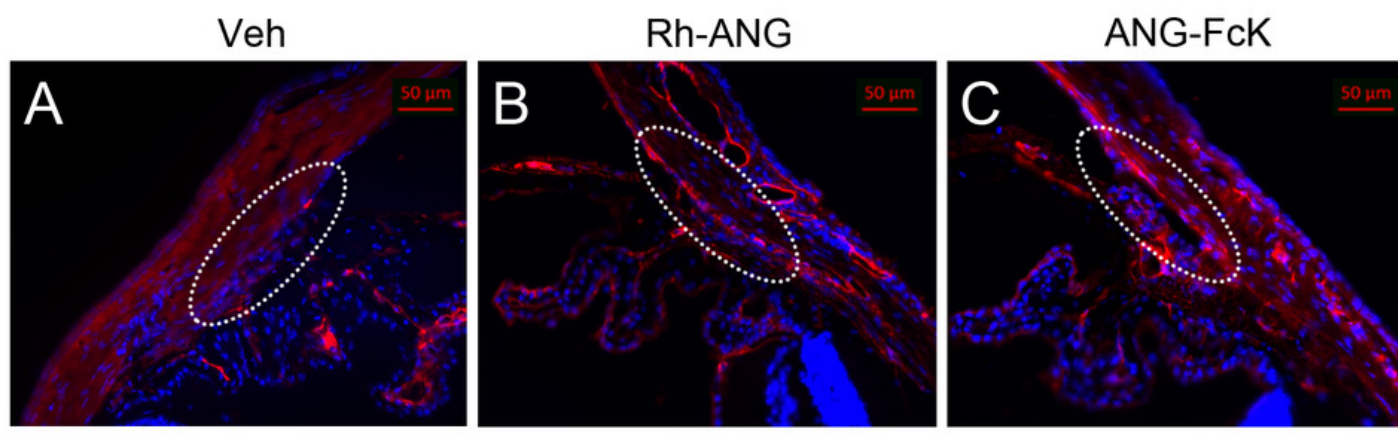

\author{
BAK (-) \\ Collagen I \\ (Red) \\ BAK (+)
}
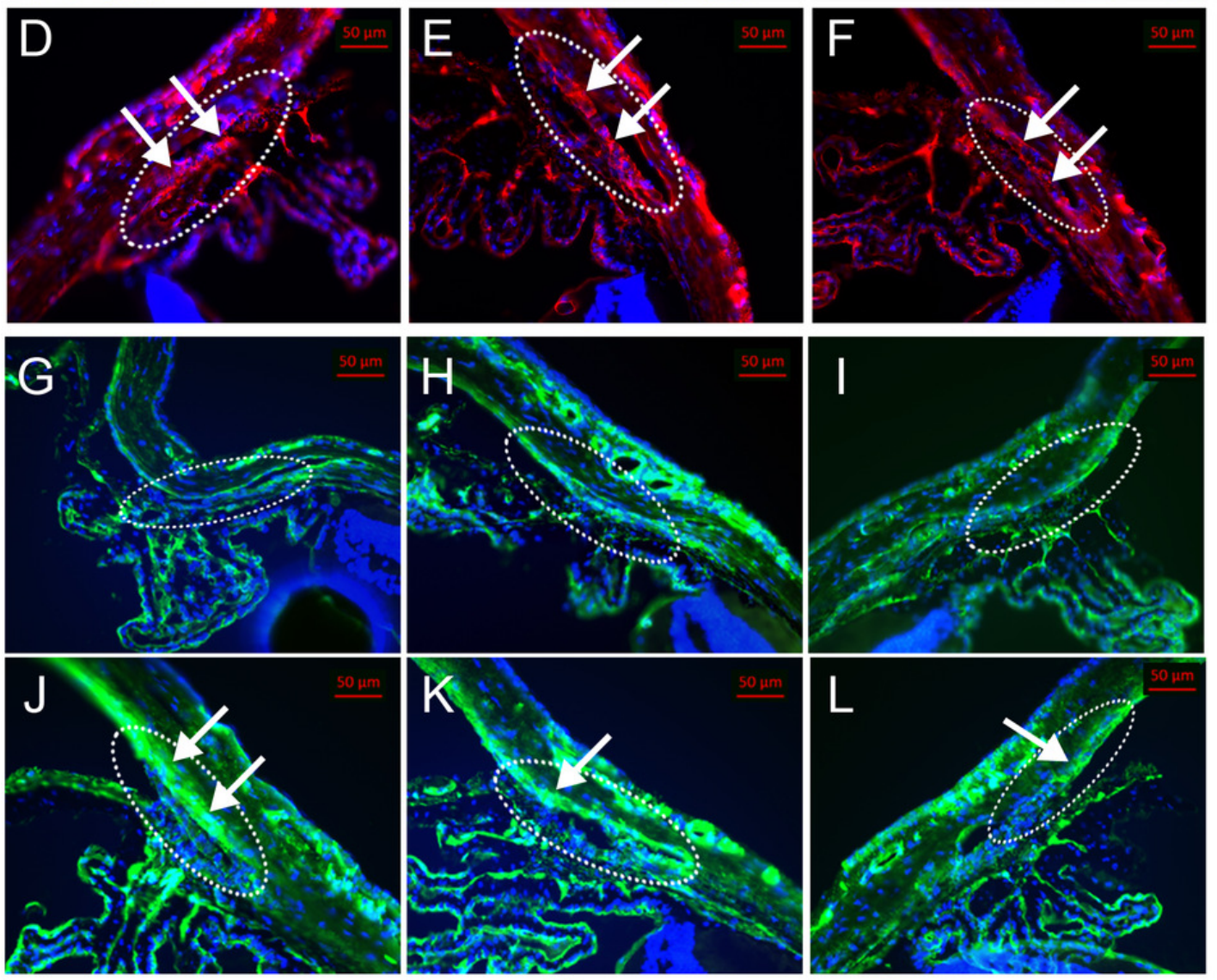

BAK (-)

Collagen IV
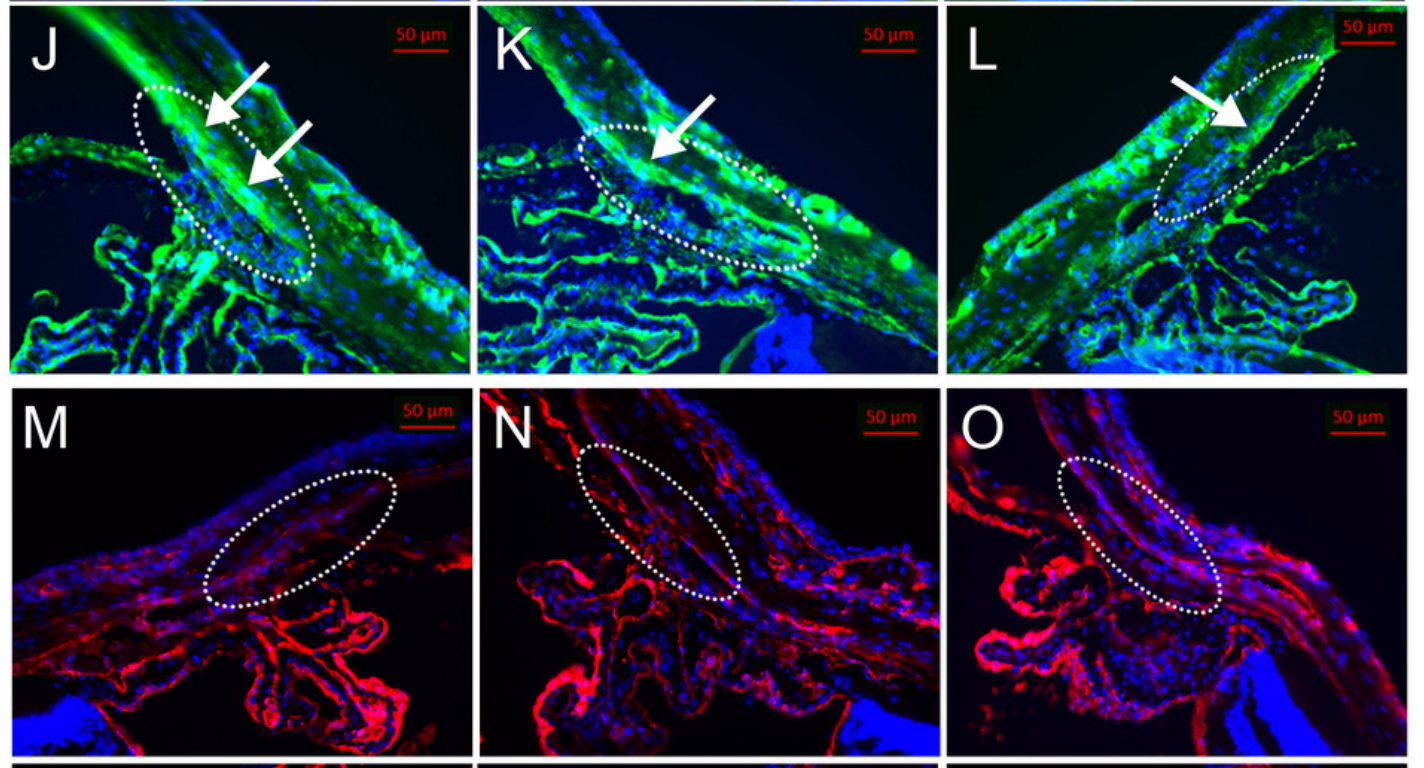

(Green)

BAK (+)
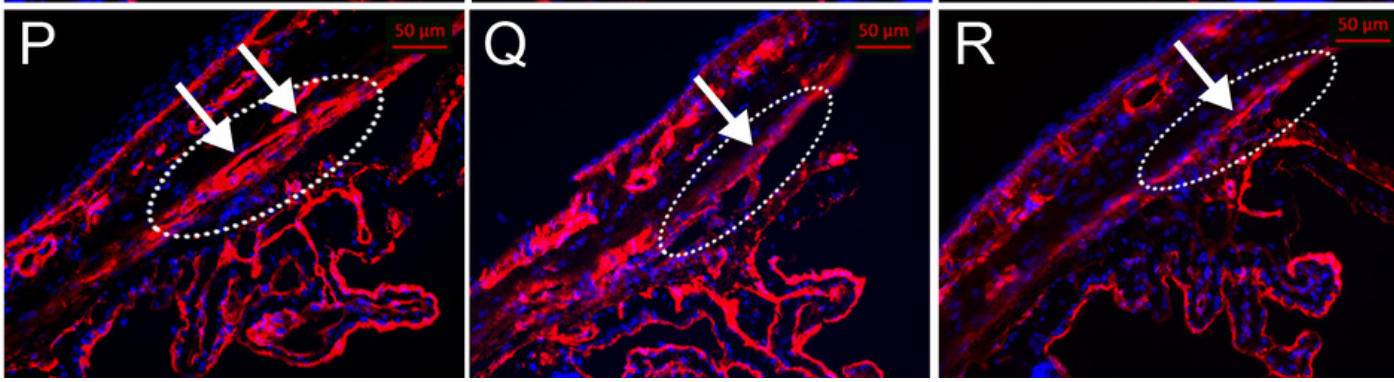

Fibronectin

(Red)

BAK (+)

PeerJ reviewing PDF | (2019:08:40251:1:2:NEW 29 Mar 2020) 


\section{Figure 4}

Immunohistochemical labeling of aqueous outflow tissues in a mouse model 02

(A) Sham-treated control. (B) Single Rh-ANG treatment. (C) Single ANG-FcK treatment. (D)

Single BAK treatment. (E) Rh-ANG with BAK treatment. (F) ANG-FcK with BAK treatment group. Staining of laminin (green) in the single BAK-treated group was more pronounced in outflow tissues (dotted oval) of the TM and the inner and outer walls of Schlemm's canal than in the Rh-ANG or ANG-FcK with BAK groups (arrowheads) but it was barely detectable in single ANG and control groups. (G) Sham-treated control. (H) Single Rh-ANG treatment. (I) Single ANG-FcK treatment. (J) Single BAK treatment. (K) Rh-ANG with BAK treatment. (L) ANG-FcK with BAK treatment group. Spotty positive $\alpha$-SMA labeling (green) in the TM layers adjacent to Schlemm's canal (arrowheads) was only detected in the eye of the single BAKtreated group, but not in any ANG-treated and control groups. (M) Sham-treated control. (N) Single Rh-ANG treatment. (O) Single ANG-FcK treatment. (P) Single BAK treatment. (Q) RhANG with BAK treatment. (R) ANG-FcK with BAK treatment group. Green fluorescent beads were deposited in outflow tissues; however, they were sparse in the single BAK-treated group. Type I collagen was used for counter-staining (red). All images were magnified 200 times. Veh $=$ vehicle for sham-treated control 


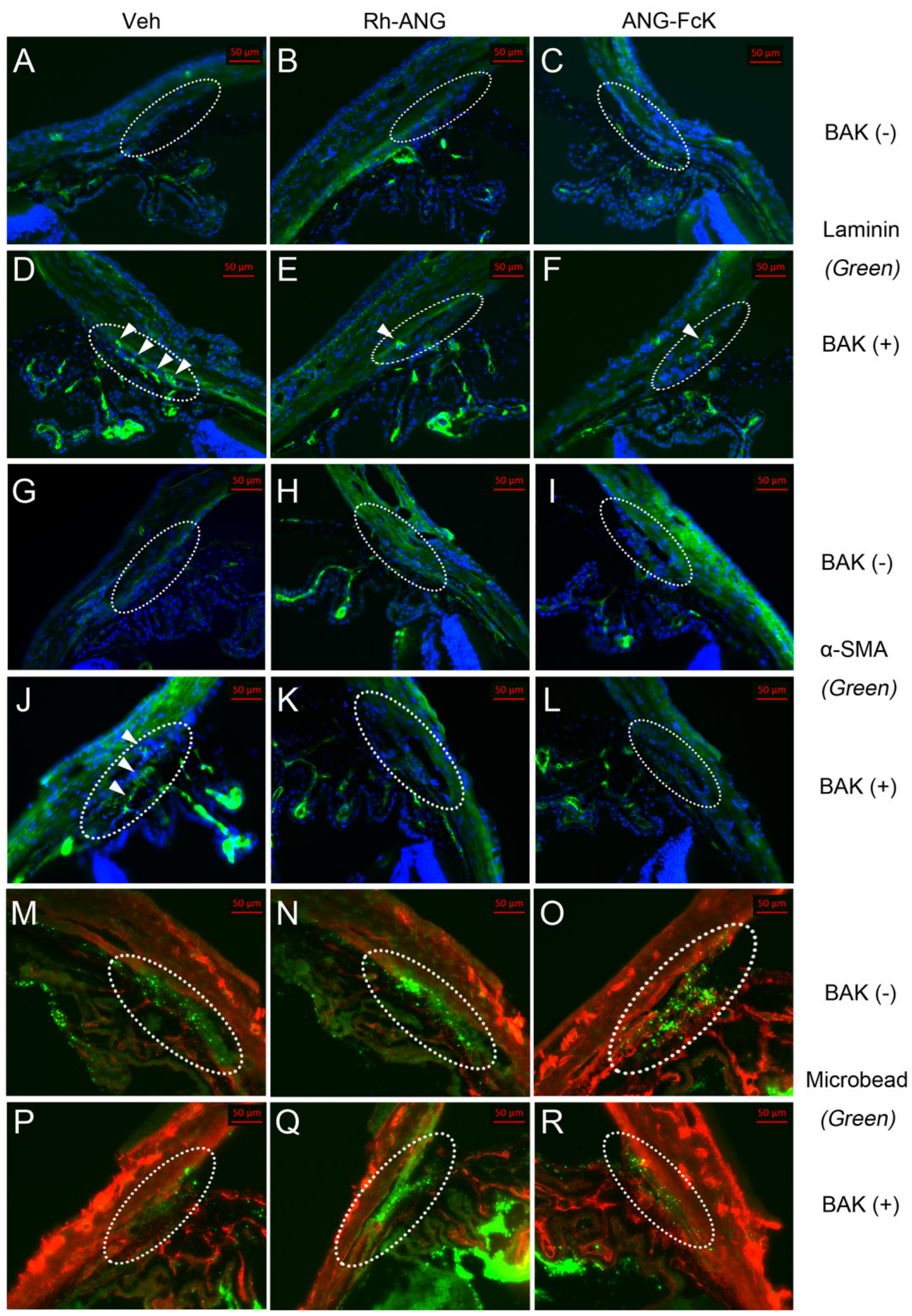

PeerJ reviewing PDF | (2019:08:40251:1:2:NEW 29 Mar 2020) 


\section{Figure 5}

Ultrastructural changes in the TM of mice treated with the combination of BAK and ANG for 4 weeks

Photographs with magnification power of 1,500 were in top row, and those with magnification power of 4,000 were in bottom row. (A) In sham-treated control mice without BAK, optically open spaces (asterisks) were often observed between juxtacanalicular connective tissue (JCT) cells. (B) Accumulation of fibrillar material within JCT was sparse and unidirectional (arrowheads). (C) In BAK-treated mice, the thickness of the lamina beam increased within the JCT (arrows). (D) The JCT was often filled with fine fibrillary material that showed an increased density and multidirectional array (empty arrowheads). Increased intracellular organelles were found in the cytoplasm of trabeculocytes with BAK treatment. (E) The ultrastructures of TM for the combination of Rh-ANG with BAK. The thickness of the lamina beam and the empty spaces within the JCT (asterisks) were preserved. (F) Fibrillar materials were denser in the combined treatment group than the control group, but sparser than in the single BAK treatment group (arrowheads) (G) The ultrastructures of TM and (H) deposition of fibrillar materials for the combination of ANG-FcK with BAK were similar with those of Rh-ANG with BAK. 

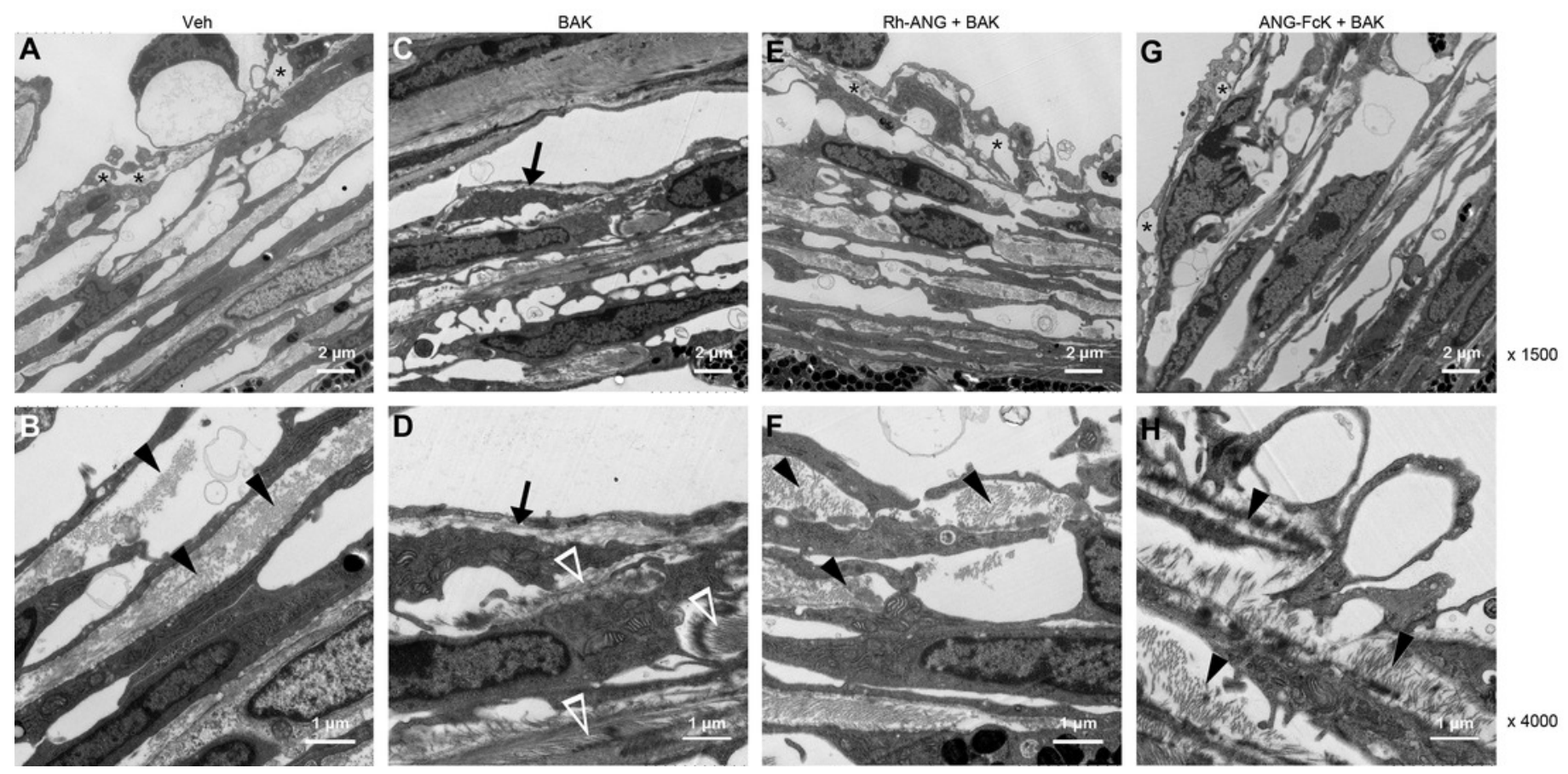